\title{
S-1 and the treatment of gastric cancer with peritoneal dissemination
}

\author{
KUNIHIKO IZUISHI $^{1,5}$, REIJI HABA ${ }^{2}$, YOSHIO KUSHIDA ${ }^{2}$, KYUICHI KADOTA ${ }^{2}$, RYUSUKE TAKEBAYASHI ${ }^{1}$, \\ TAKANORI SANO ${ }^{1,5}$, HISASHI USUKI ${ }^{1}$, MOHAMMAD AKRAM HOSSAIN ${ }^{3}$, \\ HIROHITO MORI $^{4}$, TSUTOMU MASAKI ${ }^{4}$ and YASUYUKI SUZUKI ${ }^{1}$
}

\author{
Departments of ${ }^{1}$ Gastroenterological Surgery, ${ }^{2}$ Diagnostic Pathology, ${ }^{3}$ Cell Physiology, and ${ }^{4}$ Internal Medicine \\ of Gastroenterology and Neurology, Faculty of Medicine, Kagawa University, Kagawa 761-0793; \\ ${ }^{5}$ Department of Gastroenterological Surgery, Federation of Public Services and Affiliated \\ Personnel Aid Associations, Takamatsu Hospital, Kagawa 760-0018, Japan
}

Received January 20, 2011; Accepted June 14, 2011

DOI: 10.3892/etm.2011.290

\begin{abstract}
Peritoneal dissemination is the most common metastatic pattern of gastric cancer. We frequently face the necessity for gastrectomy in the event of gastric stenosis or gastric bleeding. However, the indication for palliative gastrectomy and the effectiveness of palliative chemotherapy are not clear. We retrospectively evaluated the prognostic factors after palliative gastrectomy in 121 gastric cancer patients with peritoneal dissemination. The expression of orotate phosphoribosyl transferase (OPRT) was examined immunohistochemically. The median survival time of all patients after palliative gastrectomy was 8.8 months. In the multivariate analyses, we adjusted the data of 82 patients without liver metastases for the background of 5-fluouracil (5-FU)-based chemotherapy regimen. The analysis revealed that the degree of peritoneal dissemination (multiple vs. a few metastases or cytologypositive; $\mathrm{P}=0.01)$ and chemotherapy ( $\mathrm{S}-1$ vs. other $5-\mathrm{FU}$; $\mathrm{P}=0.01$ ) were independent predictors of survival. Particularly, $\mathrm{S}-1$ treatment was associated with a more favorable prognosis of the patients with high levels of OPRT expression compared to that of the patients with low expression. Patients with peritoneal dissemination are considered as terminal and inoperable. However, S-1 treatment may improve the survival after palliative gastrectomy in patients selected according to the degree of peritoneal dissemination and high OPRT expression.
\end{abstract}

\section{Introduction}

Peritoneal dissemination is the most common metastasis of gastric cancer. Dutch Gastric Cancer Trial reported that $26 \%$ of

Correspondence to: Dr Kunihiko Izuishi, Department of Gastroenterological Surgery, Federation of Public Services and Affiliated Personnel Aid Associations, Takamatsu Hospital, 4-18 Tenjinmae, Takamatsu, Kagawa 760-0018, Japan

E-mail: izuishi@kkr-ta-hp.gr.jp

Key words: palliative gastrectomy, S-1, gastric cancer the patients were found to have incurable cancer spread at the time of laparotomy (1). Moreover, it is the most difficult type of metastasis to treat due to an extremetly poor prognosis of 3-6 months (2). Therefore, the majority of surgeons are doubtful regarding the efficacy of surgical treatment in these types of gastric cancer cases (3). However, at times, gastrectomy must be performed for patients with far advanced gastric cancer due to gastric bleeding or gastric stenosis. Additionally, standard chemotherapies have not been established for peritoneal dissemination, and reports concerning the treatment of these cases are few. Evaluation of the effectiveness of chemotherapy is difficult, since it is not possible to detect the change in tumor size in a few millimeters of peritoneal dissemination. Instead, only measurement of the secondary changes, such as the amount of ascites, is possible. Moreover, since gastric cancer is aggressive in nature, the reduced lifespan of such patients does not allow for continued second- and third-line chemotherapies. Indeed, the outcome of treatments, such as palliative gastrectomy or chemotherapy, for peritoneal dissemination is not very encouraging $(4,5)$. It has been reported that peritonectomy and/ or intraperitoneal chemotherapy have been successful for the treatment of peritoneal dissemination. However, the survival benefits of these treatments are controversial due to the associated morbidity and low quality of life.

Recently, chemotherapy for gastric cancer has become remarkably advanced. It has been reported that S-1, an oral antitumor agent containing 5-fluouracil (5-FU), is an effective adjuvant treatment for East Asian patients after D2 gastrectomy for advanced gastric cancer (6). Furthermore, previous studies have reported that a combination chemotherapy of S-1 and cisplatin can be effective $(7,8)$. Notably, there have been several reports on the long-term survival of patients with peritoneal dissemination who received S-1 chemotherapy (9). However, S-1 chemotherapy is not always effective in all patients. To avoid unnecessary chemotherapy, it is crucial to identify specific prognostic factors that may be helpful in predicting the efficacy of chemotherapy in patients with a poor prognosis. Thymidylate synthetase (TS), which is an essential DNA de novo synthetic enzyme, is the target inhibition enzyme of the metabolite of 5-FU. Dihydropyrimidine dehydrogenase 
(DPD) is a major degrading enzyme that catabolizes 5-FU to fluorinated $\beta$-alanine and orotate phosphoribosyl transferase (OPRT) activating 5-FU and forming 5-fluoroudine monophosphate (FUMP). It has been suggested that these enzymes play a key role in the metabolic pathway of 5-FU and can be useful predictors of the patient sensitivity toward 5-FU (10-12).

In this study, we retrospectively examined the prognosis of gastric cancer patients with peritoneal dissemination after palliative gastrectomy and investigated the prognostic factors in order to determine the indications for palliative gastrectomy. This is because therapeutic planning and selection of suitable treatments is crucial in order to avoid unnecessary surgery or chemotherapy, particularly in the case of patients with an expected short lifespan.

\section{Materials and methods}

Using our database, we determined that 121 consecutive patients with peritoneal dissemination who underwent palliative gastrectomy for the treatment or prevention of gastric stenosis or bleeding were eligible for this retrospective study. The patients were treated between April 1984 and September 2008. The follow-up period was defined as the time from the day of surgery to April 2010. The inclusion criteria of patients were in accordance with the 12th edition of the Japanese General Rules for Gastric Cancer Study (13). This guideline describes the status of peritoneal dissemination in terms of three classes as follows: metastasis of the tumors to the adjacent peritoneum (P1), metastasis of a few tumors to distant peritoneum (P2), or metastasis of numerous tumors to the distant peritoneum (P3). In addition, we also included the patients with positive peritoneal washing cytology without peritoneal dissemination (P0/CY1; defined as stage 4 in the Japanese General Rules for Gastric Cancer), as many previous studies have demonstrated that $\mathrm{P} 0 / \mathrm{CY} 1$ patients have a similarly poor prognosis as patients with macroscopic peritoneal dissemination $(5,14)$.

As shown in Table I, many different types of chemotherapy were administered before 2000 since no chemotherapy had been confirmed to be particularly effective for the treatment of gastric cancer with peritoneal dissemination. Regardless of the type of chemotherapy, however, the prognosis of patients was extremely poor. It should be noted that after 2000, all patients received S-1 chemotherapy. Therefore, to analyze the efficacy of S-1 treatment as the first-line chemotherapy, we compared the survival times of patients who were administered S-1 chemotherapy and other 5-FU-based chemotherapies. S-1 treatment was administered at $60 \mathrm{mg} / \mathrm{m}^{2}$ twice daily for 4 weeks followed by a 2 -week rest period. The dosage and duration of S-1 treatment were reduced as per signs of toxicity. The chemotherapy regimens were as follows: $S-1$ therapy $(n=42)$ included $S-1$ monotherapy $(n=38), S-1$ plus low-dose cisplatin $(n=3)$ and $S-1$ plus docetaxel $(n=1)$. Other 5-FU-based chemotherapies $(n=47)$ included 5-FU ( $\mathrm{n}=2), 5-\mathrm{FU}$ plus mitomycin $\mathrm{C}(\mathrm{n}=4), 5-\mathrm{FU}$ plus cisplatin $(n=11)$, tegafur $(n=24)$ and doxifluridine $(n=6)$.

Immunohistochemistry. This study was approved by the institutional review boards of our university. DPD, TS and OPRT antibodies were kindly donated by Taiho Pharmaceutical Co., Tokushima, Japan. Briefly, the formalin-fixed, paraffinembedded gastric samples were cut into $4-\mu \mathrm{m}$ sections. The slides were incubated overnight at room temperature with
Table I. Characteristics of 121 patients with peritoneal dissemination.

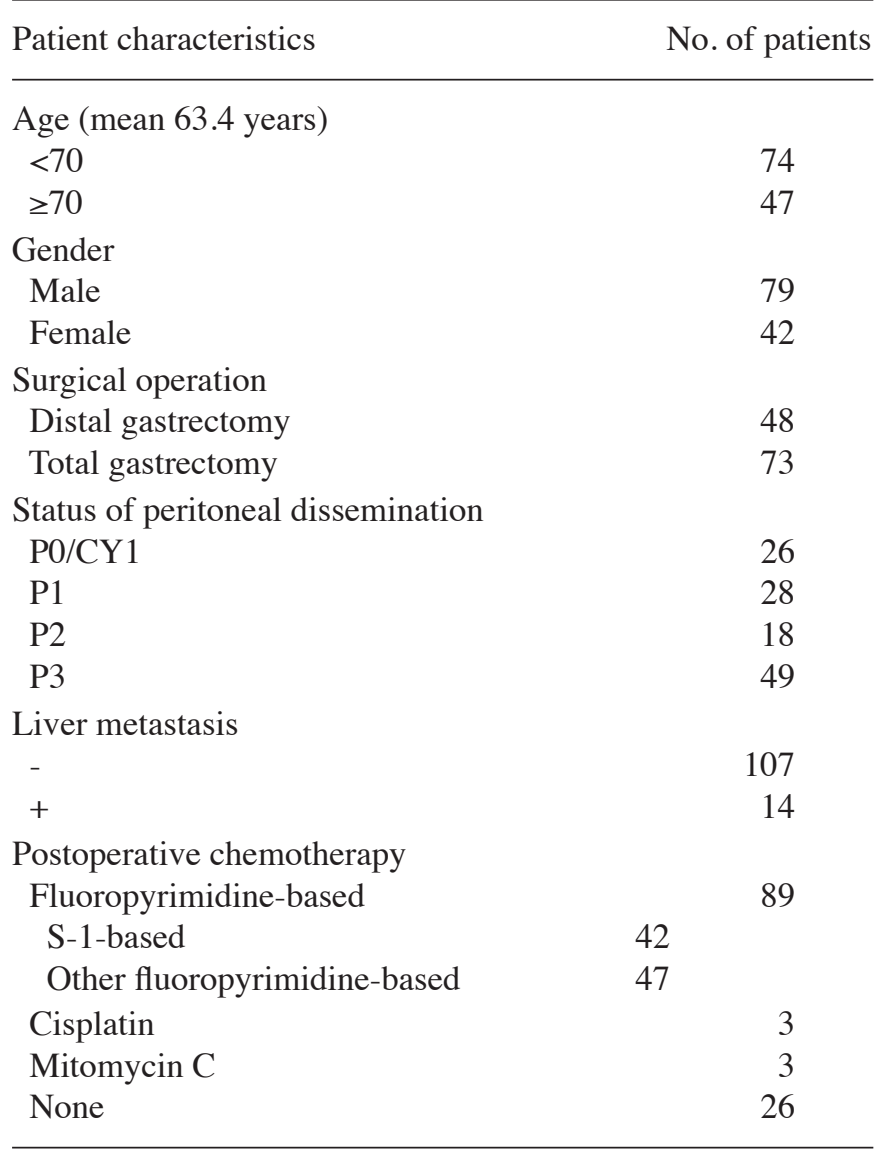

primary rabbit polyclonal antibodies for DPD, TS and OPRT (at dilutions of 1:100,1:200 and 1:100, respectively).

The immunohistochemical evaluations of DPD, TS and OPRT were reviewed by two authors (K.I. and R.H.) who had no knowledge of the patient clinical status. The expression was determined according to a previous report (12).

Statistical analysis. The differences between proportions were analyzed by the $\chi^{2}$ test. Survival curves were calculated using the Kaplan-Meier method, and a log-rank test was used to compare the survival between groups. The Cox proportional hazards model was used in multivariate survival analysis to assess the independent prognostic value of each of the variables considered. Statistical analyses were performed using SPSS software (SPSS Inc., Chicago, IL, USA). A p-value of $<0.05$ was regarded as significant.

\section{Results}

Patient characteristics. Table I shows the characteristics of the 121 patients with peritoneal dissemination. The status of the peritoneal dissemination varied ( $\mathrm{P} 0 / \mathrm{CY} 1,26$ patients; $\mathrm{P} 1$, 28 patients; P2, 18 patients; P3, 49 patients). Out of the 121 patients (79 men and 42 women) with peritoneal dissemination, 14 also had liver metastases. In all, 48 patients underwent distal gastrectomies and 73 underwent total gastrectomies. The median follow-up period for all patients was 6.7 months. The mean age of the patients was 62.7 years. 


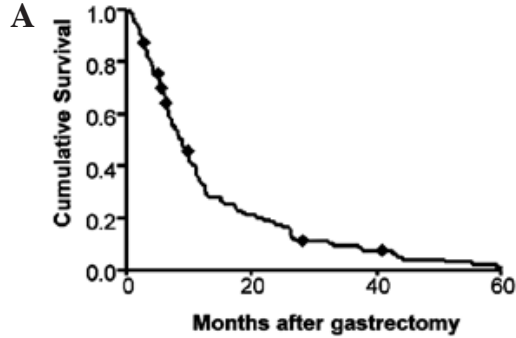

D

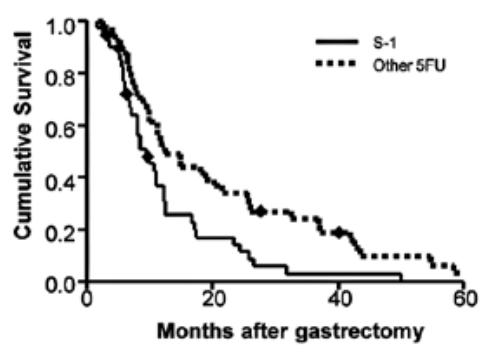

G

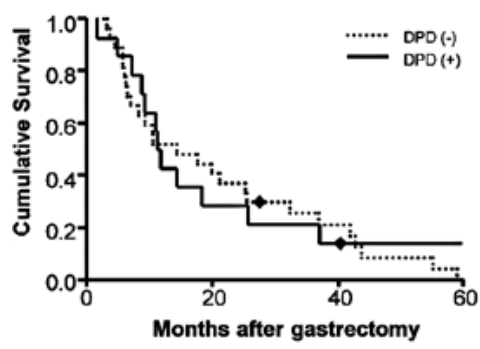

B

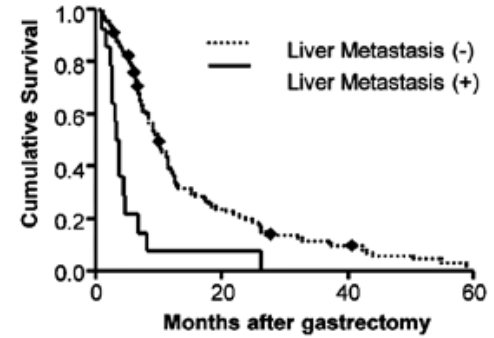

E

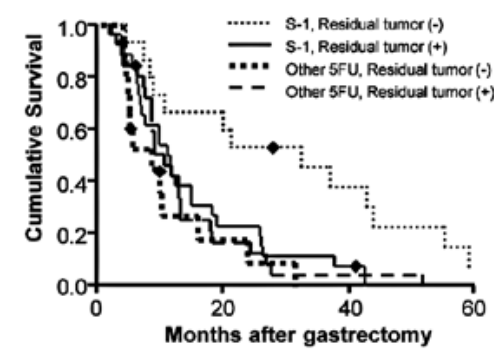

H

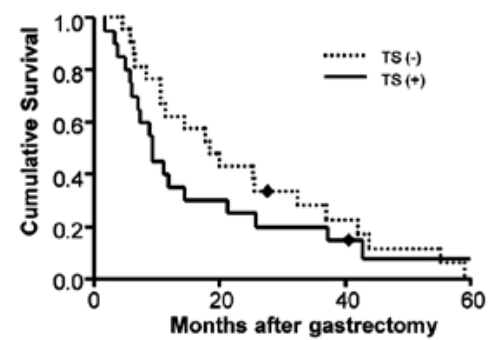

C

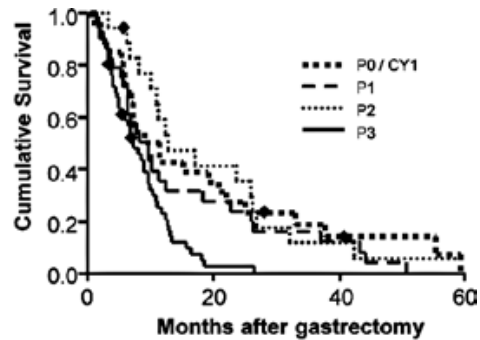

F

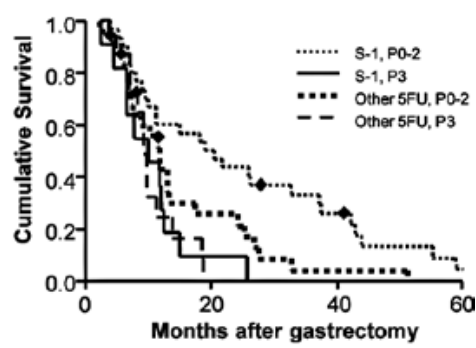

I

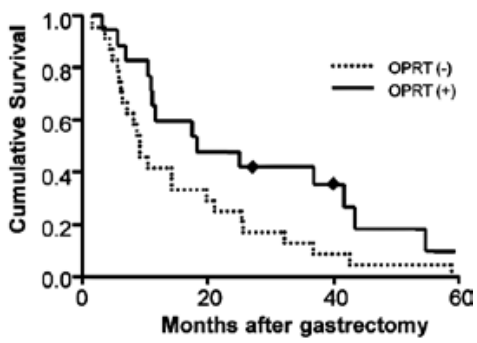

Figure 1. Kaplan-Meier survival curves for the gastric cancer patients with peritoneal dissemination. (A) The survival curve for all 121 patients shows a very poor prognosis. (B) The survival time for patients with liver metastases was significantly lower compared to that for the patients without liver metastasis $(\mathrm{P}<0.0001)$. (C) The prognosis for $\mathrm{P} 3$ patients was the poorest ( $\mathrm{P} 0 / \mathrm{CY} 1$ vs. $\mathrm{P} 3$, $\mathrm{P} 1$ vs. $\mathrm{P} 3$ and $\mathrm{P} 2$ vs. $\mathrm{P} 3$; $\mathrm{P}=0.001, \mathrm{P}=0.008$ and $\mathrm{P}<0001$, respectively). (D) Patients who received S-1 treatment survived longer than those who received other 5-FU-based chemotherapies $(\mathrm{P}=0.01)$. (E) Patients were divided into two groups according to whether or not macroscopic residual tumors were detected. The survival time improved in the $\mathrm{S}-1$ group $(\mathrm{P}=0.006)$, but a similar improvement was not noted in patients who received other 5-FU-based chemotherapies before 2000. (F) The survival time of P0-2 and P3 patients improved in the S-1 group ( $\mathrm{P}=0.003$ ), but a similar improvement was not noted in patients who received other 5-FU-based chemotherapies before 2000. (G and H) Dihydropyrimidine dehydrogenase (DPD) and thymidylate synthase (TS) expression was not significantly related to patient survival. (I) Patients with high orotate phosphoribosyl transferase (OPRT) expression lived longer compared to those with low OPRT expression. The difference among the curves was determined to be statistically significant by the log-rank test.

Overall patient survival. Fig. 1A shows the overall survival of the patients. As described in previous reports, the prognosis of patients with peritoneal dissemination who had undergone gastrectomies is extremely poor. In our study, these patients had a median survival time of 8.8 months. Furthermore, the median survival time for patients with both liver metastases and peritoneal dissemination was 3.4 months, while that for patients without liver metastases $(\mathrm{P}<0.001)$ was 9.6 months (Fig. 1B). Regarding the degrees of peritoneal dissemination (P0/CY1, P1, P2 and P3), only P3 patients demonstrated a significantly worse prognosis, and there was no statistically significant difference in the prognosis of $\mathrm{P} 0 / \mathrm{CY} 1, \mathrm{P} 1$ and $\mathrm{P} 2$ patients (Fig. 1C). Furthermore, the prognosis of $\mathrm{P} 0 / \mathrm{CY} 1$ patients was similarly as poor as for $\mathrm{P} 1$ and $\mathrm{P} 2$ patients. Therefore, in the subsequent univariate and multivariate analyses, we excluded the patients with liver metastases, and classified the four degrees of peritoneal metastasis into two categories, namely, P0-2 (which included P0/CY1, P1 and P2) and P3.

Univariate survival analysis of patients who underwent 5-FU-based chemotherapy after palliative gastrectomy. The efficacy of various types of post-operative courses, such as the best supportive care of 26 patients and the adjuvant chemotherapy of 95 patients, was examined in this study (Table I). Therefore, to evaluate the effectiveness of S-1 chemotherapy, we used patients who had undergone other types of 5-FU-based chemotherapy as a control group. Since 2000 , when S-1 became commercially available, all gastric cancer patients have been receiving S-1 chemotherapy. Therefore, the group of patients that received other 5-FU-based chemotherapies had undergone treatment before 2000. Table II shows the results of the univariate survival analyses of the clinicopathological data of 82 patients who had undergone 5-FU-based chemotherapy. Statistical differences were found with regard to the status of peritoneal dissemination, macroscopic residual cancer (except for peritoneal dissemination) and the type of chemotherapy (S-1 vs. other 5-FU-based chemotherapies). In the Cox regression multivariate analysis, macroscopic residual tumor, except for peritoneal dissemination, was not an independent prognostic factor [HR=1.20 (95\% CI 0.68-2.09)]. However, P0-2 grade of peritoneal dissemination [HR=2.14 (95\% CI 1.19-3.83)] and S-1 treatment [HR=0.59 (95\% CI 0.36-0.96)] were significant and independent predictors of good survival (Table 3). 
Table II. Univariate survival analyses of the clinicopathological data.

\begin{tabular}{|c|c|c|c|c|}
\hline Patient characteristics & No. & Median & $\mathrm{HR}(95 \% \mathrm{CI})$ & P-value \\
\hline \multicolumn{5}{|l|}{ Age (years) } \\
\hline$<70$ & 31 & 10.1 & $1.1(8.0-12.2)$ & \multirow[t]{2}{*}{0.969} \\
\hline$\geq 70$ & 51 & 11.2 & $1.2(8.9-13.5)$ & \\
\hline \multicolumn{5}{|l|}{ Gender } \\
\hline Male & 53 & 11.9 & $1.2(9.5-14.3)$ & \multirow[t]{2}{*}{0.249} \\
\hline Female & 29 & 8.9 & $2.2(4.5-13.3)$ & \\
\hline \multicolumn{5}{|l|}{ Surgical operation } \\
\hline Distal gastrectomy & 30 & 10.0 & $1.5(7.1-12.9)$ & \multirow[t]{2}{*}{0.912} \\
\hline Total gastrectomy & 52 & 11.6 & $0.8(10.0-13.3)$ & \\
\hline \multicolumn{5}{|c|}{$\begin{array}{l}\text { Macroscopic residual tumor } \\
\text { except for peritoneal dissemination }\end{array}$} \\
\hline$(-)$ & 30 & 11.2 & $5.0(1.3-21.1)$ & \multirow[t]{2}{*}{0.038} \\
\hline$(+)$ & 52 & 11.2 & $1.6(8.0-14.3)$ & \\
\hline \multicolumn{5}{|c|}{ Status of peritoneal dissemination } \\
\hline $\mathrm{P} 0-2$ & 55 & 12.5 & $2.3(8.0-17.0)$ & \multirow[t]{2}{*}{0.001} \\
\hline P3 & 27 & 8.8 & $0.5(7.8-9.9)$ & \\
\hline \multicolumn{5}{|l|}{ Tumor size (cm) } \\
\hline$<10$ & 54 & 11.2 & $1.2(8.8-13.5)$ & \multirow[t]{2}{*}{0.668} \\
\hline$\geq 10$ & 28 & 11.6 & $2.6(6.5-16.8)$ & \\
\hline \multicolumn{5}{|l|}{ Tumor type } \\
\hline Non-scirrhous type & 43 & 11.1 & $0.8(9.5-12.8)$ & \multirow[t]{2}{*}{0.141} \\
\hline Scirrhous type & 39 & 11.6 & $1.7(8.3-14.9)$ & \\
\hline \multicolumn{5}{|l|}{ ly } \\
\hline$(-)$ & 29 & 12.4 & $1.8(8.9-16.0)$ & \multirow[t]{2}{*}{0.801} \\
\hline$(+)$ & 53 & 11.1 & $1.0(9.2-13.1)$ & \\
\hline \multicolumn{5}{|l|}{$\mathrm{v}$} \\
\hline$(-)$ & 38 & 11.9 & $0.8(10.3-13.5)$ & \multirow[t]{2}{*}{0.739} \\
\hline$(+)$ & 44 & 9.5 & $1.8(5.9-13.1)$ & \\
\hline \multicolumn{5}{|l|}{ Differentiation } \\
\hline Moderate & 29 & 12.4 & $0.9(10.7-14.1)$ & \multirow[t]{2}{*}{0.646} \\
\hline Poor & 53 & 10.4 & $1.3(7.8-13.0)$ & \\
\hline \multicolumn{5}{|l|}{ T status } \\
\hline $\mathrm{T} 3$ & 68 & 11.2 & $1.0(9.2-13.2)$ & \multirow[t]{2}{*}{0.339} \\
\hline $\mathrm{T} 4$ & 14 & 7.2 & $4.0(0.0-14.9)$ & \\
\hline \multicolumn{5}{|c|}{ Postoperative chemotherapy } \\
\hline S-1 & 41 & 12.4 & $2.5(7.6-17.2)$ & \multirow[t]{2}{*}{0.010} \\
\hline Other 5-FU & 41 & 9.7 & $1.7(6.4-12.9)$ & \\
\hline
\end{tabular}

Log-rank test was applied to data of the univariate analysis. HR, hazard ratio; ly, microscopic lymphatic invasion; v, microscopic vessel invasion; T3, tumor penetration of serosa; T4, tumor invasion of adjacent structures.

Fig. 1D shows the Kaplan-Meier survival curve for the patients treated with S-1 and other 5-FU-based chemotherapies. Notably, $\mathrm{S}-1$ treatment improved the 3-year survival rates of patients $(\mathrm{P}=0.01)$ from 2.8 to $24.1 \%$. To determine which patients were actually benefited from S-1 treatment, we analyzed the patients in the macroscopic residual tumor (negative/positive) and P0-2/ P3 subgroups of the S-1 and other 5-FU-based chemotherapy groups. S-1 treatment improved the survival of patients with no macroscopic residual tumors $(\mathrm{P}=0.006)$, while other 5-FU-based treatments did not affect patient survival in this group (Fig. 1E). Similarly, S-1 treatment improved survival in the P0-2 patients $(\mathrm{P}=0.003)$, while other 5-FU-based treatments did not (Fig. 1F).
Immunohistochemistry of the 5-FU-related enzymes DPD, TS and OPRT. To further assess the group of patients with a more favorable survival (chemotherapy-sensitive patients) who underwent S-1 treatment, we examined the expression of 5-FU-related enzymes in the 41 patients of the S-1 treatment group. Immunological staining revealed that DPD, TS and OPRT were expressed in the cytoplasm. Of the 41 patients in the S- 1 treatment group, 27, 21 and 24 were negative and 14,20 and 17 were positive for the expression of DPD, TS and OPRT, respectively. Notably, DPD and TS expression was not significantly related to patient survival (Fig. 1G and H). However, patients with high OPRT expression survived longer than those with low OPRT 
Table III. Multivariate analysis of overall survival in patients who underwent 5-FU-based chemotherapy.

\begin{tabular}{lll}
\hline Factor & SE & HR (95\% CI) \\
\hline $\begin{array}{l}\text { Macroscopic residual tumor } \\
\text { except for peritoneal dissemination } \\
\quad /+\end{array}$ & 0.29 & $1.20(0.68-2.09)$ \\
$\begin{array}{l}\text { Status of peritoneal dissemination } \\
\text { P0-2/P3 }\end{array}$ & 0.30 & $2.14(1.19-3.83)$ \\
$\begin{array}{l}\text { Postoperative chemotherapy } \\
\text { S-1/Other 5-FU }\end{array}$ & 0.25 & $0.59(0.36-0.96)$ \\
\hline
\end{tabular}

The Cox regression model was applied to the multivariate analysis of overall survival. SE, standard error; HR, hazard ratio; CI, confidence interval.

expression ( $\mathrm{P}=0.043$; log-rank test). The median survival period was 19 and 10 months, respectively (Fig. 1I).

In summary, the prognosis was extremely poor for patients with peritoneal dissemination who underwent palliative gastrectomies (8.8 months). However, when patients without numerous peritoneal dissemination (non-P3 patients) and liver metastases received S-1 chemotherapy, their prognosis significantly improved (19 months). Moreover, these patients showed high OPRT expression, and their median survival time was further increased to 42.4 months (Fig. 2).

\section{Discussion}

Even though it has been over 50 years since 5-FU was developed as a chemotherapeutic agent, it remains one of the main anticancer drugs used for the treatment of a variety of different cancers. However, it has been reported that S-1, which was developed on the basis of biochemical modulation of 5-FU, has been successfully used for treating gastric cancer. The response rate of S-1 monotherapy in phase II trials was more than $40 \%$ in patients with advanced or recurrent gastric cancer $(15,16)$. The Adjuvant Chemotherapy Trial of S-1 for Gastric Cancer (ACTS-GC) showed that single-agent S-1 adjuvant chemotherapy for stage II/III gastric cancer patients who underwent curative D2 surgery improved the survival rates of the patients significantly (6). The 3 -year overall survival rate was $80.1 \%$ in the S-1 group and $70.1 \%$ in the surgery-only group. Notably, the peritoneum relapse rate was significantly decreased to 11.2 from $15.8 \%$ with S-1 treatment. Nevertheless, S-1 treatment did not significantly inhibit hematogenous relapse $(11.3 \%, \mathrm{~S}-1$ group; $10.2 \%$, surgery-only group). The chemotherapy for peritoneal dissemination is usually difficult, as the cancerous area is isolated from the blood flow and the peritoneal blood barrier prevents drug distribution $(17,18)$. It has been reported that after S-1 treatment, the concentration of 5-FU rises four times higher in intraperitoneal tumors and six times higher in ascites as compared to its concentration after other 5-FU treatments; this may help explain the greatly improved survival times after S-1 treatment (19,20). In addition, 5-chloro-2,4-dihydroxypyridine (CDHP), a specific DPD inhibitor used in S-1 treatment, also plays an important role with regard to modulating the concentration of 5-FU. Generally, $80-90 \%$ of the 5-FU is rapidly catabolized by DPD in the liver $(21,22)$, but pharmacokinetic studies have indicated that the addition of CDHP results in a 20 -fold increase in the serum concentration of 5-FU.

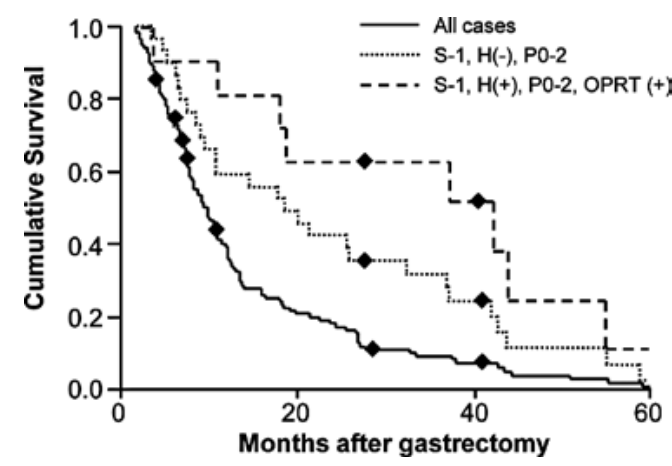

Figure 2. Survival curves of patients selected according to the degree of peritoneal dissemination (PD) and OPRT expression. The 3-year survival of all patients, patients treated with $\mathrm{S}-1$ without liver metastasis and multiple PD, and high-OPRT patients treated with S-1 without liver metastasis and multiple PD, were increased by $9.6,33.3$ and $63.3 \%$, respectively.

In this study, we evaluated the prognostic factors after palliative resection in patients with peritoneal dissemination. Our results indicate that the median survival time of patients with both peritoneal dissemination and liver metastases is only 3.4 months. The Dutch Gastric Cancer Group has suggested that palliative gastrectomies are beneficial when the tumor extension is limited to a single site; widespread tumor growth, peritoneal, liver or lymph node (1). However, since the majority of patients with peritoneal dissemination in this study had not undergone gastrectomies, the survival times of the patients with peritoneal dissemination were not specifically examined. Indeed, the relative benefits of performing palliative gastrectomies on patients with peritoneal dissemination remain debatable. A previous study found that half the patients with M1 gastric cancer (74\% of those with peritoneal dissemination) who did not undergo gastrectomy required subsequent intervention, while the other half did not require any further treatment. Only $12 \%$ of the patients required surgical treatment (23), since percutaneous endoscopic gastrostomy for gastric outlet obstruction, radiation therapy and endoscopic laser therapy for bleeding were useful intervention techniques in other patients. Therefore, they concluded that surgical approaches, including palliative resection, were unnecessary. However, all of the patients died within 3 years.

On the other hand, several studies have shown that certain patients have relatively good prognoses, and palliative resection is associated with increased survival times in P1 or P2 
patients $(1,24)$. Our results also showed that when patients with peritoneal dissemination were divided into two groups, the prognosis for $\mathrm{P} 0-2$ patients was much better than that for P3 patients. Therefore, it can be considered that $\mathrm{P} 0-2$ patients may be good candidates for palliative gastrectomies. More importantly, it should be noted that $\mathrm{S}-1$ treatment prolonged the survival of $\mathrm{P} 0-2$ patients or patients with no macroscopic residual tumor; this may not have been apparent prior to the clinical introduction of S-1 therapy.

However, sensitivity to anticancer agent, 5-FU, varies from patient to patient. In our analysis, OPRT was a significant prognostic indicator of survival. DPD is mainly distributed in the liver, while OPRT is distributed in both the tumor and liver (22) and is known to metabolize 5-FU at both these sites. It has been reported that the growth inhibition caused by $5-\mathrm{FU}$ is closely correlated with the levels of OPRT mRNA in cancer cell lines. Moreover, down-regulation of OPRT by small interfering RNA decreased the sensitivity of the tumor cells to 5-FU in vitro (25). Several clinical studies have suggested that TS, DPD and OPRT expression in gastric and colorectal cancers is associated with 5-FU sensitivity (10-12).

In this study, comparison of S-1 data with other 5-FU data was conducted based on the consecutive recruitment of two groups of patients at the same hospital. However, this study has several limitations. The design involved the collection of patient data over a long period and dealt with variable types of regimens. However, as mentioned by Sakuramoto et al, the effectiveness of specific regimens was not yet verified in large clinical trials before S-1; nevertheless, many chemotherapeutic regimens have been reported for the treatment of gastric cancer (6). Particularly, this issue has not yet been reported in peritoneal dissemination. In addition, when we compared P3 patients as the internal standard, the survival curve was identical to those of the other 5-FU groups. Therefore, it is unlikely that the long period of data collection and the variable types of 5-FU chemotherapies were responsible for the difference we observed between the two groups in this study.

In conclusion, the development of strategies for increasing the survival times of patients with peritoneal dissemination is a challenge in gastric cancer treatment. Our result does not recommend palliative gastrectomy for all gastric cancer patients with gastric bleeding or obstruction. It presents the contraindication of palliative gastrectomy. The candidates for gastrectomy should be chosen on the basis of the degree of peritoneal dissemination and high OPRT expression. Unfortunately, the period of survival is reduced in far advanced gastric cancer patients, therefore, we must keep in mind the indication for palliative gastrectomy.

\section{References}

1. Hartgrink HH, Putter H, Klein Kranenbarg E, Bonenkamp JJ and van de Velde CJ: Value of palliative resection in gastric cancer. Br J Surg 89: 1438-1443, 2002.

2. Ishizone S, Maruta F, Saito H, Koide N, Sugiyama A, Nakayama J and Miyagawa S: Efficacy of S-1 for patients with peritoneal metastasis of gastric cancer. Chemotherapy 52: 301-307, 2006.

3. Sadeghi B, Arvieux C, Glehen O, et al: Peritoneal carcinomatosis from non-gynecologic malignancies: results of the EVOCAPE 1 multicentric prospective study. Cancer 88: 358-363, 2000.

4. Kodera Y, Yamamura Y, Shimizu Y, et al: Peritoneal washing cytology: prognostic value of positive findings in patients with gastric carcinoma undergoing a potentially curative resection. J Surg Oncol 72: 60-64, 1999.
5. Bando E, Yonemura Y, Takeshita Y, et al: Intraoperative lavage for cytological examination in 1,297 patients with gastric carcinoma. Am J Surg 178: 256-262, 1999.

6. Sakuramoto S, Sasako M, Yamaguchi T, et al: Adjuvant chemotherapy for gastric cancer with S-1, an oral fluoropyrimidine. N Engl J Med 357: 1810-1820, 2007.

7. Kubota T: The role of S-1 in the treatment of gastric cancer. Br J Cancer 98: 1301-1304, 2008.

8. Koizumi W, Narahara H, Hara T, et al: S-1 plus cisplatin versus S-1 alone for first-line treatment of advanced gastric cancer (SPIRITS trial): a phase III trial. Lancet Oncol 9: 215-221, 2008.

9. Kobayashi O, Konishi K, Kanari M, et al: Unusual survival for more than 2 years with peritoneal metastases of gastric cancer. Gastric Cancer 5: 47-50, 2002.

10. Kai K, Kitajima Y, Hiraki M, et al: Quantitative double-fluorescence immunohistochemistry (qDFIHC), a novel technology to assess protein expression: a pilot study analyzing 5-FU sensitive markers thymidylate synthase, dihydropyrimidine dehydrogenase and orotate phosphoribosyl transferases in gastric cancer tissue specimens. Cancer Lett 258: 45-54, 2007.

11. Ishikawa M, Miyauchi T and Kashiwagi Y: Clinical implications of thymidylate synthetase, dihydropyrimidine dehydrogenase and orotate phosphoribosyl transferase activity levels in colorectal carcinoma following radical resection and administration of adjuvant 5-FU chemotherapy. BMC Cancer 8: 188, 2008.

12. Tokunaga Y, Sasaki H and Saito T: Clinical role of orotate phosphoribosyl transferase and dihydropyrimidine dehydrogenase in colorectal cancer treated with postoperative fluoropyrimidine. Surgery 141: 346-353, 2007.

13. Japanese Gastric Cancer Association: Japanese Classification of Gastric Carcinoma, 2nd English edition. Gastric Cancer 1: 10-24, 1998.

14. Bonenkamp JJ, Songun I, Hermans J and van de Velde CJ: Prognostic value of positive cytology findings from abdominal washings in patients with gastric cancer. Br J Surg 83: 672-674, 1996.

15. Sakata Y, Ohtsu A, Horikoshi N, Sugimachi K, Mitachi Y and Taguchi T: Late phase II study of novel oral fluoropyrimidine anticancer drug S-1 (1 M tegafur- $0.4 \mathrm{M}$ gimestat-1 M otastat potassium) in advanced gastric cancer patients. Eur J Cancer 34: 1715-1720, 1998.

16. Koizumi W, Kurihara M, Nakano S and Hasegawa K: Phase II study of S-1, a novel oral derivative of 5-fluorouracil, in advanced gastric cancer. Oncology 58: 191-197, 2000.

17. Esquivel J, Vidal-Jove J, Steves MA and Sugarbaker PH: Morbidity and mortality of cytoreductive surgery and intraperitoneal chemotherapy. Surgery 113: 631-636, 1993.

18. Jacquet $P$ and Sugarbaker PH: Peritoneal-plasma barrier. Cancer Treat Res 82: 53-63, 1996.

19. Mori T, Fujiwara Y, Yano M, Tamura S, Yasuda T, Takiguchi S and Monden M: Experimental study to evaluate the usefulness of S-1 in a model of peritoneal dissemination of gastric cancer. Gastric Cancer 6 (Suppl 1): 13-18, 2003.

20. Yamagata S, Nakata B and Hirakawa K: Dihydropyrimidine dehydrogenase inhibitory fluoropyrimidine S-1 may be effective against peritoneal dissemination in gastric cancer. Oncol Rep 12: 973-978, 2004

21. Ichikawa W and Sasaki Y: Challenges in predicting the clinical outcome in S-1-based chemotherapy for gastric cancer patients. Int J Clin Oncol 13: 206-211, 2008.

22. Kuramochi H, Hayashi K, Uchida K, et al: 5-Fluorouracil-related gene expression levels in primary colorectal cancer and corresponding liver metastasis. Int J Cancer 119: 522-526, 2006.

23. Sarela AI, Miner TJ, Karpeh MS, Coit DG, Jaques DP and Brennan MF: Clinical outcomes with laparoscopic stage M1, unresected gastric adenocarcinoma. Ann Surg 243: 189-195, 2006.

24. Saito H, Yamada Y, Tsujitani S and Ikeguchi M: Clinicopathologic characteristics of gastric cancer patients who underwent noncurative gastrectomy with long-term survival. Langenbecks Arch Surg 394: 99-103, 2009.

25. Sakamoto E, Nagase H, Kobunai T, Oie S, Oka T, Fukushima M and Oka T: Orotate phosphoribosyltransferase expression level in tumors is a potential determinant of the efficacy of 5-fluorouracil. Biochem Biophys Res Commum 363: 216-222, 2007. 